\title{
Pulmonary function deficits in newborn screened infants with cystic fibrosis managed with standard UK care are mild and transient
}

\author{
Gwyneth Davies ${ }^{1,2}$, Janet Stocks ${ }^{1}$, Lena P. Thia ${ }^{1}$, Ah-Fong Hoo ${ }^{1,2}$, \\ Andrew Bush ${ }^{3,4}$, Paul Aurora ${ }^{1,2}$, Lucy Brennan ${ }^{1}$, Simon Lee ${ }^{1}$, Sooky Lum¹, \\ Philippa Cottam ${ }^{1}$, Joanne Miles ${ }^{2}$, Jane Chudleigh ${ }^{5,6}$, Jane Kirkby ${ }^{1,2}$, Ian \\ M. Balfour-Lynn ${ }^{3,4}$, Siobhán B. Carr ${ }^{3,7}$, Colin Wallis ${ }^{2}$, Hilary Wyatt ${ }^{5}$ and \\ Angie Wade $\mathbb{1}^{8}$ on behalf of the London Cystic Fibrosis Collaboration (LCFC) ${ }^{9}$
}

\begin{abstract}
Affiliations: ${ }^{1}$ Respiratory, Anaesthesia and Critical Care Section, Infection, Immunity, Inflammation Programme, UCL Great Ormond Street Institute of Child Health, London, UK. ${ }^{2}$ Respiratory Medicine, Great Ormond Street Hospital for Children NHS Foundation Trust, London, UK. ${ }^{3}$ Dept of Paediatric Respiratory Medicine, Royal Brompton Hospital, London, UK. ${ }^{4}$ National Heart and Lung Institute, Imperial College London, London, UK. ${ }^{5}$ Dept of Child Health, King's College London, London, UK. ${ }^{6}$ Dept of Child Health, City, University of London, London, UK. ${ }^{7}$ Dept of Paediatric Respiratory Medicine, Royal London Hospital, London, UK. ${ }^{8}$ Clinical Epidemiology, Nutrition and Biostatistics, Population, Policy and Practice Programme, UCL Great Ormond Street Institute of Child Health, London, UK. ${ }^{9}$ A full list of the LCFC members and their affiliations can be found in the Acknowledgements section.
\end{abstract}

Correspondence: Gwyneth Davies, Respiratory, Critical Care and Anaesthesia Section, Infection, Immunity, Inflammation Programme, UCL Great Ormond Street Institute of Child Health, 30 Guilford Street, London WC1N 1EH, UK. E-mail: gwyneth.davies@ucl.ac.uk

@ERSpublications

Lung function changes in newborn screened infants with cystic fibrosis are mild and transient up to age 2 years http://ow.ly/7gkW30etHtb

Cite this article as: Davies G, Stocks J, Thia LP, et al. Pulmonary function deficits in newborn screened infants with cystic fibrosis managed with standard UK care are mild and transient. Eur Respir J 2017; 50: 1700326 [https://doi.org/10.1183/13993003.00326-2017].

ABSTRACT With the advent of novel designer molecules for cystic fibrosis (CF) treatment, there is huge need for early-life clinical trial outcomes, such as infant lung function (ILF). We investigated the degree and tracking of ILF abnormality during the first 2 years of life in CF newborn screened infants.

Forced expiratory volume in $0.5 \mathrm{~s}$ (FEV0.5), lung clearance index (LCI) and plethysmographic functional residual capacity were measured at $\sim 3$ months, 1 year and 2 years in 62 infants with CF and 34 controls.

By 2 years there was no significant difference in FEV0.5 z-score between $\mathrm{CF}$ and controls, whereas mean LCI z-score was 0.81 (95\% CI 0.45-1.17) higher in CF. However, there was no significant association between LCI z-score at 2 years with either 3-month or 1-year results. Despite minimal average group changes in any ILF outcome during the second year of life, marked within-subject changes occurred. No child had abnormal LCI or FEV 0.5 on all test occasions, precluding the ability to identify "high-risk" infants in early life.

In conclusion, changes in lung function are mild and transient during the first 2 years of life in newborn screened infants with CF when managed according to a standardised UK treatment protocol. Their potential role in tracking disease to later childhood will be ascertained by ongoing follow-up. 


\section{Introduction}

The advent of gene mutation class-specific therapies for cystic fibrosis (CF) and demonstration of their efficacy in older children and adults has led to demands to introduce them in infancy, before significant lung damage occurs. However, if such trials are to be undertaken, highly sensitive trial end-points are needed to monitor both for efficacy and safety during this vulnerable period of rapid lung growth. A recent European Cystic Fibrosis Society Clinical Trial Network consensus concluded that infant lung function (ILF) testing using the raised volume rapid thoracic compression (RVRTC) technique should not be used as a primary outcome in clinical trials of infants with CF until further evidence is available [1]. Similar recommendations have been made with respect to infant multiple breath washout (MBW) [2].

The London Cystic Fibrosis Collaboration (LCFC; www.ucl.ac.uk/london-cystic-fibrosis) is following-up a newborn screened (NBS) CF cohort and contemporaneous controls. We have reported previously that at 3 months, forced expiratory volume in $0.5 \mathrm{~s}$ (FEV0.5) was reduced in 25\% (17 out of 68) of infants, while lung clearance index (LCI) was elevated in $21 \%$ (15 out of 70) [3]. ILF at 1 year was predicted by ILF measured at 3 months and FEV0.5 improved over the first year of life with standard UK treatment [4]. We suggested that those infants with abnormal ILF (e.g. an elevated LCI) in early life may comprise a subgroup in whom specific treatment interventions would make the greatest difference. Yet if innovative therapies with the potential to modify disease are to be deployed, there must be both clinical need and the ability to assess outcome. If our observed improvement in ILF was sustained between 1 and 2 years with standard treatment alone in NBS infants, this would have significant implications for clinical trial design involving novel treatments for CF in early life, when the developing lung is potentially at its most vulnerable [5].

The aim of this observational study was to investigate the tracking of ILF in the first 2 years of life in CF NBS infants managed with standard UK therapy, and to consider the implications of this in relation to the use of such outcomes as end-points in clinical trials conducted over this time period. Based on our previous findings at 1 year, we hypothesised that group stability of ILF would be maintained to 2 years in NBS CF infants. Some results presented here have been published as abstracts [6-8].

\section{Material and methods}

Full details of both the recruitment of the LCFC cohort of infants with CF diagnosed by NBS and contemporaneous healthy controls, and the prospective observational study design have been published $[3,4]$. In brief, NBS CF infants born between January 2009 and July 2011 who were referred to the six specialist CF centres in the LCFC were eligible for recruitment. Healthy controls were recruited contemporaneously from Homerton University Hospital (London, UK). Infants were ineligible if born at $<36$ weeks' gestation or had coexisting congenital abnormalities. The study was approved by the North Thames multicentre research ethics committee (\#09/HO71/314). Informed written parental consent was obtained. Participating centres prospectively completed case record forms at diagnosis and at each subsequent clinic visit. CF infants were started on multivitamins and vitamin E, pancreatic enzyme replacement therapy where appropriate and, in accordance with UK CF Trust guidelines, oral prophylactic flucloxacillin, according to a standardised treatment protocol [4]. All subjects in the LCFC NBS CF cohort attending between 1.5 and 2.4 years with a previous visit at 3 months and/or 1 year were included in this 2 -year follow-up. Subjects were free from acute respiratory tract infection for $\geqslant 3$ weeks prior to testing. MBW, plethysmography and RVRTC were performed on each test occasion at the ILF laboratory at University College London Great Ormond Street Institute of Child Health (London, UK), as described previously [3, 4]. The main ILF outcomes were LCI, plethysmographic functional residual capacity (FRCpleth) and FEV0.5. Oral sedation (chloral hydrate $60-100 \mathrm{mg} \cdot \mathrm{kg}^{-1}$; maximum $1000 \mathrm{mg}$ ) was administered prior to each occasion. MBW results were analysed using custom-made software (P. Gustafsson; version 2012). ILF data were electronically exported to a research database (Rebase software; Rebase, London, UK) in which data for each child were linked according to test occasion with demographic and clinical information. ILF

Support statement: This study is supported by grants from the UK Cystic Fibrosis Trust; Special Trustees: Great Ormond Street Hospital for Children; Smiths Medical; Comprehensive Local Research Network; and supported by the National Institute for Health Research (NIHR) Biomedical Research Centre at Great Ormond Street Hospital for Children NHS Foundation Trust and University College London Great Ormond Street Institute of Child Health. G. Davies was supported by a NIHR academic clinical lectureship at University College London. A. Bush is an NIHR senior investigator and was supported by the NIHR Respiratory Disease Biomedical Research Unit at the Royal Brompton and Harefield NHS Foundation Trust and Imperial College London. Funding information for this article has been deposited with the Crossref Funder Registry.

This article presents independent research funded by the NIHR. The views expressed are those of the authors and not necessarily those of the NHS, the NIHR or the Department of Health.

Conflict of interest: Disclosures can be found alongside this article at erj.ersjournals.com 
outcomes were converted to $\mathrm{z}$-scores using published reference equations derived from healthy infants and young children using identical equipment and protocols [9-11]. Clinical information was collected at routine clinical visits and at each test occasion, including history of intravenous antibiotics and airway microbiology results (from cough swabs or from bronchoalveolar lavage at 1 year).

\section{Statistical analysis}

Differences between control and CF groups at each test occasion were compared using unpaired t-tests. We planned to study at least 60 NBS CF children and 30 healthy controls at $\sim 2$ years of age to provide $80 \%$ power to detect differences of $\geqslant 0.725 \mathrm{z}$-scores in the three primary ILF outcomes ( $90 \%$ power to detect differences of $0.825 \mathrm{z}$-scores) at the $5 \%$ significance level.

Individual line plots over time were used to illustrate change within-subject between tests. Paired t-tests were used to quantify average change between any two test occasions within groups and the standard deviation to quantify variability of those changes. Unpaired t-tests were used to compare change over time between groups. To further investigate tracking of ILF in children with CF, abnormalities on each test occasion were defined as $>1.96 \mathrm{z}$-scores for LCI and FRCpleth or $<-1.96 \mathrm{z}$-scores for FEV0.5.

Regression was used to investigate any relationship between change in ILF and interval between tests. To investigate change over time according to disease status and treatment severity, CF subjects were grouped according to whether they had received intravenous antibiotics or isolated relevant pathogens (Pseudomonas aeruginosa, Staphylococcus aureus or Haemophilus influenzae) in respiratory culture by the time of their test at 2 years. Sample estimates (differences between groups and changes over time) are presented with $95 \%$ confidence intervals to facilitate interpretation.

\section{Results}

62 NBS CF infants and 34 healthy controls with prior ILF results had tests repeated at 2 years of age. The study population is summarised in online supplementary table E1. The proportion of subjects with technically satisfactory ILF results at each test occasion ranged from $77 \%$ to $98 \%$ (online supplementary table E2). Results presented here focus on the relationship between ILF results at 1 and 2 years, as tracking between 3 months and 1 year has been published [4].

\section{Cross-sectional data}

Cross-sectional results for both CF and healthy infants at 2 years are summarised in table 1, with results at 3 months and 1 year in online supplementary table E3. At 1 year, there were significant differences between $\mathrm{CF}$ and control groups for all three primary lung function outcomes (online supplementary table E3b). However, by 2 years, there was no significant difference in FEV0.5 between CF and controls. Over the same time period, differences between CF and controls increased slightly by $0.12 \mathrm{z}$-scores for LCI, with a mean $(95 \% \mathrm{CI})$ difference of $0.81(0.45-1.17) \mathrm{z}$-scores between groups by 2 years (figure 1 and table 1 ),

\begin{tabular}{|c|c|c|c|c|}
\hline & Cystic fibrosis & Healthy controls & Difference $(95 \% \mathrm{CI})$ & p-value \\
\hline Subjects $\mathrm{n}$ & 62 & 34 & & \\
\hline Age at test, weeks & $95.0 \pm 7.3$ & $96.2 \pm 7.7$ & $-1.24(-4.39-1.92)$ & 0.44 \\
\hline zHeight & $0.48 \pm 1.00$ & $0.71 \pm 1.18$ & $-0.23(-0.69-0.22)$ & 0.31 \\
\hline zWeight & $0.24 \pm 0.92$ & $0.40 \pm 0.86$ & $-0.16(-0.54-0.22)$ & 0.41 \\
\hline zBMI & $-0.07 \pm 0.92$ & $-0.03 \pm 0.90$ & $-0.04(-0.42-0.35)$ & 0.85 \\
\hline zLCI & $0.78 \pm 0.93$ & $-0.03 \pm 0.64$ & $0.81(0.45-1.17)$ & 0.001 \\
\hline zFRCpleth & $0.85 \pm 1.30$ & $0.16 \pm 1.28$ & $0.69(0.11-1.26)$ & 0.02 \\
\hline zFEV 0.5 & $-0.35 \pm 1.00$ & $-0.14 \pm 1.23$ & $-0.21(-0.71-0.29)$ & 0.41 \\
\hline zFVC & $-0.19 \pm 0.94$ & $0.04 \pm 0.92$ & $-0.23(-0.66-0.21)$ & 0.30 \\
\hline zFEF $25-75 \%$ & $-0.41 \pm 0.95$ & $-0.24 \pm 1.15$ & $-0.17(-0.64-0.3)$ & 0.48 \\
\hline
\end{tabular}

Data are presented as mean $\pm \mathrm{SD}$, unless otherwise stated. For numbers of subjects with each outcome at each test occasion see online supplementary table E2. Although not primary outcomes, forced vital capacity (FVC) and forced expiratory flow at $25-75 \%$ of $\mathrm{FVC}(\mathrm{FEF} 25-75 \%)$ results are included in this table to allow comparison with the published literature. z: z-score; BMI: body mass index; LCI: lung clearance index; FRCpleth: plethysmographic functional residual capacity; FEV 0.5 : forced expiratory volume in $0.5 \mathrm{~s}$. 

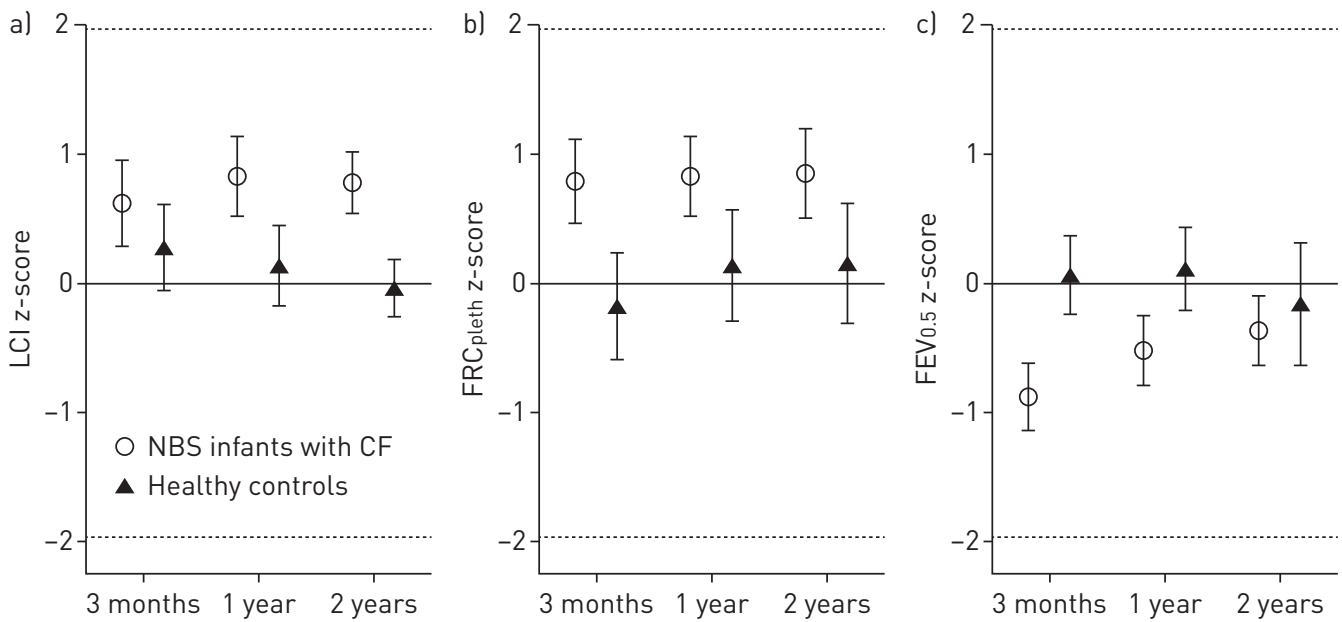

FIGURE 1 Lung function across the first 2 years of life in healthy control infants and infants with cystic fibrosis (CF). Data are presented as mean $(95 \% \mathrm{CI}) \mathrm{z}$-scores at the 3-month, 1-year and 2-year test occasions for a) lung clearance index (LCI); b) plethysmographic functional residual capacity (FRCpleth); and c) forced expiratory volume in $0.5 \mathrm{~s}(\mathrm{FEV} 0.5)$. Limits of normality are represented by the dashed lines at $\pm 1.96 \mathrm{z}$-scores. More detailed results, together with the comparison between cystic fibrosis and control subjects at each time point are presented in table 1 and online supplementary table E3. NBS: newborn screening.

whereas difference in FRCpleth z-score remained stable. The impact of using recently updated published RVRTC reference equations is summarised in the online supplementary material.

\section{Change over time}

There was no significant group change over time for any ILF outcome in controls. In contrast, as reported previously [4], there was a significant improvement in the FEV0.5 in CF infants, particularly in the first year of life (online supplementary table E4). A comparison of changes in lung function during the second year of life between CF and controls is summarised in table 2. While mean changes in ILF were minimal during the second year of life, the relatively wide standard deviations reflect marked within-subject change between test occasions.

\section{"Abnormal" lung function}

The proportion of CF infants with "normal" or "abnormal" results for each ILF outcome at each test occasion is shown in table 3 . At the time of the 2-year test, abnormal results were only detected in $15 \%$ (nine out of 61) of CF infants for LCI, 19\% (11 out of 57) for FRCpleth and 7\% (four out of 56) for FEV 0.5. No child had abnormalities in all three outcomes on any test occasion. No child had an abnormal LCI or FEV0.5 on all test occasions, and only two (5\%) out of $44 \mathrm{CF}$ infants had an abnormal FRCpleth on all test occasions.

TABLE 2 Comparison of changes in lung function and nutritional outcomes over time in infants with cystic fibrosis $(\mathrm{CF})$ and healthy controls $(\mathrm{HC})$ during the second year of life

\begin{tabular}{lccc} 
& CF & HC & Difference (95\% Cl) CF-HC \\
\hline Test interval, weeks & $42.2 \pm 7.8$ & $42.8 \pm 8.2$ & $-0.6(-4.1-2.8)$ \\
$\Delta$ zHeight & $-0.04 \pm 0.47$ & $-0.05 \pm 0.54$ & $0.01(-0.2-0.22)$ \\
$\Delta$ zWeight & $-0.08 \pm 0.46$ & $-0.05 \pm 0.43$ & $-0.03(-0.22-0.16)$ \\
$\Delta$ zBMI & $-0.10 \pm 0.61$ & $-0.06 \pm 0.78$ & $-0.05(-0.34-0.24)$ \\
$\Delta$ zLCI & $0.00 \pm 1.37$ & $-0.15 \pm 0.83$ & $0.15(-0.39-0.69)$ \\
$\Delta$ zFRCpleth & $0.02 \pm 1.13$ & $0.01 \pm 1.10$ & $0.01(-0.5-0.52)$ \\
$\Delta$ zFEV .5 & $0.19 \pm 1.03$ & $-0.20 \pm 1.19$ & $0.39(-0.12-0.90)$
\end{tabular}

Data are presented as the mean \pm SD change in $z$-score $(\Delta z)$ for each outcome, between testing at $\sim 1$ year and $\sim 2$ years, unless otherwise stated. For numbers of subjects with each outcome, see online supplementary table E2. Similar comparisons of changes between 3 months and 1 year, and 3 months and 2 years are shown in online supplementary table E5. BMI: body mass index; LCl: lung clearance index; FRCpleth: plethysmographic functional residual capacity; FEV0.5: forced expiratory volume in $0.5 \mathrm{~s}$. 
TABLE 3 Proportion of newborn screened cystic fibrosis infants with "normal" or "abnormal" results on each test occasion, and on more than one test occasion

3 months 1 year 2 years 3 months and 1 year 3 months and 2 years 1 year and 2 years All test occasions

\begin{tabular}{|c|c|c|c|c|c|c|c|}
\hline Total LCl & 57 & 59 & 61 & 54 & 56 & 58 & 53 \\
\hline Normal LCI & $48(84)$ & 49 (83) & $52(85)$ & $41(76)$ & $39(70)$ & $41(71)$ & $34(64)$ \\
\hline Abnormal LCI & $9(16)$ & $10(17)$ & $9(15)$ & $5(9)$ & $1(2)$ & $2(3)$ & $0(0)$ \\
\hline Total FRCpleth & 50 & 59 & 57 & 47 & 47 & 54 & 44 \\
\hline Abnormal FRCpleth & $9(18)$ & $10(17)$ & $11(19)$ & $4(9)$ & $4(9)$ & $3(6)$ & $2(5)$ \\
\hline Total FEV 0.5 & 59 & 58 & 56 & 56 & 53 & 54 & 52 \\
\hline Normal FEV0.5 & $51(86)$ & 54 (93) & 52 (93) & $46(82)$ & $43(81)$ & $46(85)$ & 39 (75) \\
\hline All normal ${ }^{\#}$ & $27(57)$ & $36(63)$ & $35(65)$ & $23(52)$ & $14(34)$ & 20 (39) & $12(31)$ \\
\hline All abnormal & $0(0)$ & $0(0)$ & $0(0)$ & $0(0)$ & $0(0)$ & $0(0)$ & $0(0)$ \\
\hline
\end{tabular}

Data are presented as $\mathrm{n}$ or $\mathrm{n}(\%)$. LCl: lung clearance index; FRCpleth: plethysmographic functional residual capacity; FEV0.5: forced expiratory volume in $0.5 \mathrm{~s}$. " : "all" represents LCl, FRCpleth and FEV0.5; "abnormal" and "normal" defined on basis of 1.96 z-score threshold for LCl and FRCpleth and -1.96 for FEV 0.5 .

The association of results between different test occasions within each of the primary ILF outcomes is shown in online supplementary figure E1. In contrast to the highly significant relationship in both FRCpleth and FEV 0.5 across all test occasions in infants with CF, LCI at 2 years was not predicted by LCI measured at either 3 months or 1 year. Of the $10 \mathrm{CF}$ infants with an abnormal LCI at 1 year, all but two had a result within the normal range by $\sim 2$ years (online supplementary figure E1). Similarly, only two out of nine infants with abnormal LCI at 2 years also had an abnormal 1-year result.

\section{Clinical status}

Line plots demonstrating change in LCI z-score over time at the individual level are shown in figure 2, with CF infants separated according to $P$. aeruginosa status. Similar plots for other ILF outcomes are shown in online supplementary figure E2, along with a comparison of results according to whether infants had ever received intravenous antibiotics. Considerable within-subject change in LCI occurred between tests, even in health, and the magnitude of such change was not related to either the isolation of P. aeruginosa or treatment with intravenous antibiotics by the final ILF test in those with CF (figure 2, online supplementary figure E2 and table E6). In addition, the lack of relationship between the magnitude or direction of change in LCI with respect to $P$. aeruginosa status or treatment with intravenous antibiotics is illustrated in online supplementary figure E3. A similar pattern was observed for FRCpleth and FEV0.5, with the exception of the greater improvement in FEV0.5 during the first year of life in infants in whom P. aeruginosa was not isolated by their final ILF test (online supplementary table E6). Similarly, the magnitude or direction of change between tests was not related to whether or not infants had isolated either S. aureus or H. influenzae, or any major CF pathogen (P. aeruginosa, S. aureus or H. influenzae) by 2 years (data not shown). Results of regression analysis revealed no significant association between magnitude of within-subject, between-test change in ILF and interval between tests.

\section{Discussion}

\section{Summary of main findings}

We report for the first time that in a NBS CF cohort treated with UK standardised therapy, there was no significant difference in FEV0.5 between healthy controls and CF infants by 2 years of age. In contrast to reports by the Australian Respiratory Early Surveillance Team for CF (AREST-CF) [12], NBS CF babies in our cohort did not experience deteriorating lung function over the first 2 years of life. While mean LCI $z$-score and FRCpleth z-score were significantly higher in CF infants than controls at 2 years, neither increased significantly during the second year of life. Those individuals who did exhibit abnormal ILF at any point often reverted to normality on subsequent testing, suggesting that during infancy such changes are reversible rather than progressive. Within our NBS CF cohort we could not identify any individual with consistently abnormal LCI or FEV0.5 who could thus be preferentially selected to receive novel therapies. In contrast to the relationship between 3-month and 1-year results previously reported in this cohort [4], there was no significant association between LCI z-score at 2 years and that at either 3 month or 1 year. 

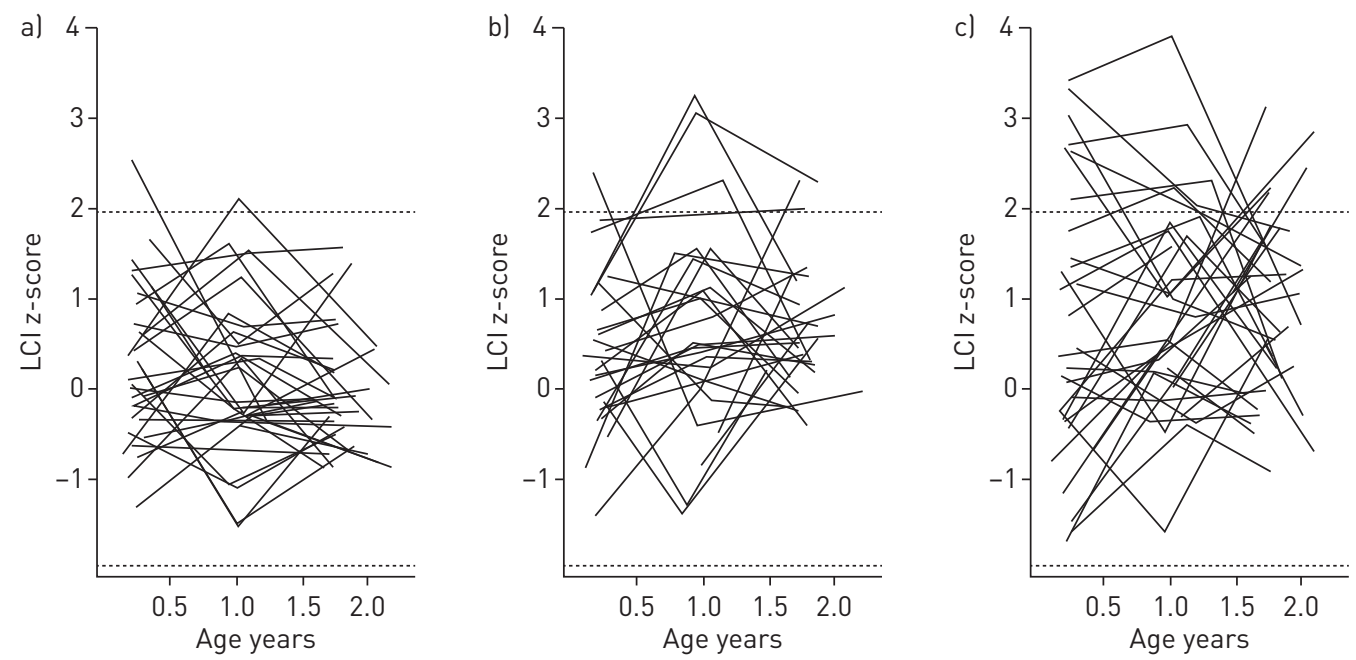

FIGURE 2 Comparison of within-subject change for lung clearance index (LCI) over the first 2 years of life in a) healthy controls; b) cystic fibrosis (CF) infants without Pseudomonas aeruginosa; c) CF infants in whom $P$. aeruginosa had been isolated on at least one occasion prior to their 2-year infant lung function test. Each subject is represented by an individual line. Z-scores for $\mathrm{LCl}$ are plotted against age at lung function test. Limits of normality are represented by the dashed lines at \pm 1.96 z-scores. Infants with CF are separated according to $P$. aeruginosa status at the time of their 2-year infant lung function test. Similar plots for plethysmographic functional residual capacity and forced expiratory volume in $0.5 \mathrm{~s}$ and for change over time according to whether the child had received intravenous antibiotics by their 2-year test are presented in online supplementary figure E2.

\section{Strengths and limitations}

The strengths of this study include prospective recruitment of the LCFC NBS cohort and contemporaneous controls, allowing unique insights into the impact of CF on early-life pulmonary function. Without controls, an understanding of change over time would be challenging. Results were interpreted using z-scores from recently published reference equations, derived using identical equipment and methodology [9-11]. In addition, the inclusion of a control group, who were similar in terms of body size, ensured that any changes in the physiological dead space to tidal volume ratio over the study period would not influence interpretation of LCI results. The equipment and techniques for measuring ILF remained standardised and constant throughout the study period, as did the sedation protocol. Technical success rates for ILF tests in our NBS LCFC cohort continue to be better than other longitudinal studies involving infants with CF (as summarised by the European CF Society Clinical Trial Network) [1], particularly when compared with those that involved testing across multiple centres [13].

A range of ILF tests were performed to reflect the various aspects of pathophysiology most commonly reported in CF lung disease, with the primary outcomes of LCI, FRCpleth and FEV0.5 selected a priori to avoid any risk of data dredging or misinterpretation due to use of an excessive number of measurements. We have not reported the tidal breathing ratio, having previously determined that it is not useful in identifying diminished airway function in infants with $\mathrm{CF}$, nor respiratory rate, which is poorly predictive of diminished airway function in this population [14]. While rarely reported, between-test variability within our control group is in keeping with that in older children, variability of up to $1.2 \mathrm{z}$-scores in FEV1 being recorded in children aged 5-11 year over the course of a year [15]. Using an observational study design, DAVIs et al. [13] did not recommend inclusion of FEV0.5 or FRCpleth measured during infancy as a primary efficacy end-point in clinical trials due to within-subject variability between tests, technical challenges and the requirement for large sample sizes to detect efficacy.

Judgements regarding the clinical usefulness of any biomarker requires an assessment of its reliability, validity and responsiveness [16]. However, objective assessments of surrogate measures such as lung function require a different approach, particularly when such measurements are being undertaken in infants. Sedation is required for most infant lung function tests, the administration of which is not advised either in the presence of an exacerbation or at frequent intervals. Furthermore, sedation may cause at least temporary disruption of sleep patterns and the duration of these tests (up to 3-4 $\mathrm{h}$ including the period to induce sleep) can place a real burden on parents if they are expected to attend for follow-up visits at intervals of $<6$ months, a factor which would reduce compliance in any clinical trial. It is therefore extremely difficult to use ILF tests to reliably assess acute response to either exacerbations or treatment. However, by undertaking repeated measurements at $\sim 9$-monthly intervals during periods of clinical 
stability, information regarding the magnitude of any changes and extent to which ILF tracks during the first 2 years of life in NBS infants with CF managed on standard care can be obtained, knowledge of which is vital if planning to use such tests in future clinical trials.

In addition to natural variability observed in health, improvement of lung function by the age of 2 years following earlier "abnormalities" in CF infants could reflect treatment intensification in the interim, either in response to symptomatic deterioration or following the identification of pathogens such as $P$. aeruginosa. The grouping of infants into those that had and had not isolated $P$. aeruginosa or been treated with intravenous antibiotics was simply to reflect relative disease burden, rather than an attempt to relate ILF to specific clinical events or interventions. Although our study was not designed to determine the dynamic effect of exacerbations or treatment response on ILF, neither the isolation of P. aeruginosa nor treatment with intravenous antibiotics by 2 years of age was associated with the magnitude of ILF variability over this period. However, the influence of early-life exacerbations on childhood lung function may become detectable at subsequent follow-up [17].

Conclusions from a study such as this might differ in regions with differing treatment protocols, prevalence of CF gene mutations or modifier genes, or environmental exposures. The antibiotic protocols used were defined primarily to standardise care between the LCFC centres in line with current UK practice rather than reflecting any evidence that antibiotic prophylaxis would impact favourably (or otherwise) on ILF outcomes. Ideally, study clinicians would have remained blinded to results, but this was not considered to be ethical in an observational study such as this. While this could be viewed as a potential weakness, given the complete overlap in direction and variability of change in ILF between those with and without prior intravenous antibiotics or CF pathogens such as P. aeruginosa, this is unlikely to have influenced our findings. In our study, $P$. aeruginosa had been isolated on at least one occasion in 32 (52\%) patients by the time of the 2-year test, although only four infants had any evidence of chronic infection. The relatively high frequency of "P. aeruginosa ever" by 2 years of age is in keeping with recent reports and reflects the fact that this cohort were under close surveillance, $P$. aeruginosa being isolated not only from the bronchoalveolar lavage at 1 year, but also from regular cough swabs throughout the study period. However, we accept that diagnosis of lower respiratory infection status in infants is difficult, and incorrect classification remains a possibility.

We performed high resolution computed tomography scans (HRCT) at 1 year of age, but the changes were so mild, and the variability between observers consequently so great [18], that we did not feel justified ethically in repeating the scan at 2 years unless clinically indicated for an individual; the continued relative normality of lung function is supportive of that decision.

\section{Clinical significance}

Our study design was a pragmatic means of informing future clinical trials in that it provides evidence both on the magnitude of ILF abnormalities that might be expected on standard therapy, as well as the extent to which such changes track during the first 2 years of life, in exactly the group of infants who might be recruited to an intervention study. Without observational data it would be difficult to design a study to evaluate effects of any novel medium-term interventions. This evidence is strengthened by our ability to interpret results in relation to the normal variability that occurs in health, since we not only had a contemporaneous control group, but also reference equations derived using identical methods and equipment. The clinical importance of ILF tests in CF lung disease will be clarified by the continuing follow-up of this cohort into the preschool years [19], which will establish whether there is evidence of tracking over a longer time period. Although mean LCI was static over the second year of life in our cohort, the small increase in $\mathrm{z}$-scores in comparison to controls may represent a degree of deterioration at the group level, which may become more apparent with subsequent follow-up. The importance of physiological outcomes during the preschool years is already recognised, as results can reflect subsequent status at school age [20], and a recent study has confirmed that LCI is a useful marker to track early disease progression in preschool children with CF [21].

It is possible to demonstrate clinical efficacy of treatment interventions assessed according to functional trial end-points even when baseline results are within the normal range, as illustrated by results from the phase III clinical trial of the cystic fibrosis transmembrane regulator potentiator ivacaftor in children aged 6-11 years with a G551D mutation [22]. However, in contrast to older children, or possibly a clinically diagnosed infant cohort, interventions aimed at reducing the rate of decline (rather than improvement) in FEV would not be appropriate in an infant NBS population such as ours, since FEV0.5 improved to normal levels during the first 2 years of life with standard UK care alone. While attempts could be made to diminish the mild elevations of LCI or FRCpleth observed in CF infants by the age of 2 years, the transient nature of within-subject abnormalities at 3 months or 1 year in infants treated with UK standard care alone calls into question the clinical relevance of this approach. 
As previously reported for our CF NBS cohort, 1-year ILF was predicted by results obtained from testing at 3 months [4]. However, the combination of relatively normal results at 2 years and marked bidirectional within-subject change over time (online supplementary figure E2), challenge the value of modelling ILF outcomes at 2 years. Therefore, we analysed results at 2 years with respect to those at 3 months and 1 year separately, rather than in a repeated measures analysis. Any long-term value of ILF in terms of predicting later CF disease status will become clearer as our current cohort is followed-up through the preschool years and beyond. The transient nature of the observed changes in lung function in our cohort is encouraging, but does mean that our previous proposal to try to identify a "high-risk" subgroup of NBS CF infants based on "abnormalities" at either 3 months or 1 year of age is not feasible.

\section{Comparison with other studies}

To our knowledge, the only other prospective longitudinal study reporting ILF outcomes in NBS infants with CF is that from the AREST-CF group. Although both AREST-CF and the LCFC detected deficits in ILF by $\sim 3$ months in such infants $[3,12,23]$, results from these two studies are widely discrepant at later time points. Instead of improvement to 1 year and maintenance of ILF to 2 years as reported here, lung function appeared to deteriorate significantly over this period in the AREST-CF infants (mean \pm SD FEV0.5 $\mathrm{Z}$-score $-1.4 \pm 1.2,-2.4 \pm 1.1$ and $-4.3 \pm 1.6$ at $\sim 5$ months, $\sim 1$ year and $\sim 2$ years of age, respectively) [12]. However, of note was the conversion of RVRTC outcomes to z-scores using historical control data collected with different equipment [24], and the absence of a contemporaneous control group within the AREST-CF study. Possible explanations for the differences between results from these two NBS cohorts have recently been summarised by BuSH and SLY [25]. Interestingly, continued longitudinal follow-up of the AREST-CF cohort has reported much better lung function at school age than might be expected from the infant results [26]. Although our results contrast with those reported by AREST-CF, DAVIs et al. [27] also reported normal FEV0.5 yet high within-subject change between ILF tests in CF infants participating in their inhaled hypertonic saline clinical trial. Continued follow-up of both the LCFC and AREST-CF cohorts will provide crucial insights to the natural history of CF lung disease and allow evaluation of proposed predictors of abnormal lung function or structural changes on chest HRCT in later childhood.

\section{Conclusion}

In contrast to previous studies from AREST-CF, we show that lung function as assessed by measurements of LCI, FRCpleth and FEV0.5 is well preserved to 2 years of age in our cohort of NBS infants with CF managed with UK standard care. The transient nature of any abnormalities observed during this time period suggests that such changes may remain reversible during early life. Whether these results can be translated to other CF populations, such as infants not receiving prophylactic antibiotic therapy or undergoing regular surveillance by infant lung function, is unclear. Nevertheless, the relative normality of infant lung function up to 2 years of age in this prospectively followed cohort of NBS CF infants managed with standard UK care is encouraging.

\section{Acknowledgements}

We thank the infants and parents who participated in this study, and gratefully acknowledge contributions by all members of the London NBS CF Collaboration (Ah-Fong Hoo, Ammani Prasad (UCL Great Ormond Street Institute of Child Health and Great Ormond Street Hospital for Children), Andrew Bush, Angie Wade, Anu Shankar (University Hospital Lewisham and St George's Hospital), Catherine Owens (UCL Great Ormond Street Institute of Child Health and Great Ormond Street Hospital for Children), Caroline Pao (Royal London Hospital), Colin Wallis, Deeba Ahmed (UCL Great Ormond Street Institute of Child Health), Gary Ruiz (King's College Hospital), Hilary Wyatt, Ian Balfour-Lynn, Jane Chudleigh (King's College Hospital), Jane Davies (Royal Brompton Hospital and National Heart and Lung Institute, Imperial College), Janet Stocks (director), John Price (King's College Hospital), Lena Thia, Lucy Brennan, Mark Rosenthal (Royal Brompton Hospital), Paul Aurora, Ranjan Suri (Great Ormond Street Hospital for Children), Richard Chavasse (St George's Hospital), Siobhan Carr, Sooky Lum, The Thanh Diem Nguyen (UCL Great Ormond Street Institute of Child Health) and Gwyneth Davies) and Per Gustafsson (Central Hospital, Skövde, Sweden) for ongoing advice and support with respect to multiple breath washout by mass spectrometry.

Author contributions: J. Stocks, A. Bush, A. Wade and I.M. Balfour-Lynn were responsible for the conception and design of the study. J. Stocks and A-F. Hoo were responsible for supervision of the study, and together with J. Chudleigh, for research governance issues including ethics committee approval. Infants with cystic fibrosis were recruited by the paediatric respiratory consultants participating in the LCFC, including A. Bush, I.M. Balfour-Lynn, S.B. Carr, H. Wyatt, C. Wallis and P. Aurora. A-F. Hoo, J. Chudleigh, L.P. Thia and L. Brennan recruited the healthy infants, A-F. Hoo, J. Miles, L. Brennan and L.P. Thia undertook lung function measurements and analysis. G. Davies, J. Kirkby, S. Lum and J. Stocks calculated and interpreted lung function results. P. Cottam, S. Lee and S. Lum managed the research database. G. Davies and A. Wade performed statistical analyses. G. Davies, J. Stocks, A. Wade and A. Bush drafted the manuscript; all remaining authors revised and approved the manuscript for intellectual content before submission. 


\section{References}

1 Matecki S, Kent L, de Boeck K, et al. Is the raised volume rapid thoracic compression technique ready for use in clinical trials in infants with cystic fibrosis? J Cyst Fibros 2016; 15: 10-20.

2 Subbarao P, Milla C, Aurora P, et al. Multiple-breath washout as a lung function test in cystic fibrosis. A Cystic Fibrosis Foundation workshop report. Ann Am Thorac Soc 2015; 12: 932-939.

3 Hoo AF, Thia LP, Nguyen TT, et al. Lung function is abnormal in 3-month-old infants with cystic fibrosis diagnosed by newborn screening. Thorax 2012; 67: 874-881.

4 Nguyen TT, Thia LP, Hoo AF, et al. Evolution of lung function during the first year of life in newborn screened cystic fibrosis infants. Thorax 2014; 69: 910-917.

5 Stocks J, Hislop A, Sonnappa S. Early lung development: lifelong effect on respiratory health and disease. Lancet Respir Med 2013; 1: 728-742.

6 Brennan LC, Thia LP, Hoo AF, et al. Evolution of lung function during the first two years of life in infants with cystic fibrosis diagnosed by newborn screening. Thorax 2013; 68: Suppl. 3, A6-A7.

7 Thia LP, Hoo AF, Brennan L, et al. Stable lung function is maintained over 2 years in newborn screened (NBS) CF infants. Eur Respir J 2013; 42; Suppl. 57: 1072s.

8 Davies G, Thia L, Hoo AF, et al. Within-subject variability of lung function in newborn screened (NBS) CF infants. Eur Respir J 2016; 48: Suppl. 60, PA4864.

9 Lum S, Stocks J, Stanojevic S, et al. Age and height dependence of lung clearance index and functional residual capacity. Eur Respir J 2013; 41: 1371-1377.

10 Nguyen TT, Hoo AF, Lum S, et al. New reference equations to improve interpretation of infant lung function. Pediatr Pulmonol 2013; 48: 370-380.

11 Lum S, Bountziouka V, Wade A, et al. New reference ranges for interpreting forced expiratory manoeuvres in infants and implications for clinical interpretation: a multicentre collaboration. Thorax 2016; 71: $276-283$.

12 Pillarisetti N, Williamson E, Linnane B, et al. Infection, inflammation, and lung function decline in infants with cystic fibrosis. Am J Respir Crit Care Med 2011; 184: 75-81.

13 Davis SD, Rosenfeld M, Kerby GS, et al. Multicenter evaluation of infant lung function tests as cystic fibrosis clinical trial endpoints. Am J Respir Crit Care Med 2010; 182: 1387-1397.

14 Ranganathan SC, Goetz I, Hoo AF, et al. Assessment of tidal breathing parameters in infants with cystic fibrosis. Eur Respir J 2003; 22: 761-766.

15 Kirkby J, Bountziouka V, Lum S, et al. Natural variability of lung function in young healthy school children. Eur Respir J 2016; 48: 411-419.

16 De Boeck K, Kent L, Davies J, et al. CFTR biomarkers: time for promotion to surrogate end-point. Eur Respir J 2013; 41: 203-216.

17 Byearnes CA, Vidmar S, Cheney JL, et al. Prospective evaluation of respiratory exacerbations in children with cystic fibrosis from newborn screening to 5 years of age. Thorax 2013; 68: 643-651.

18 Thia LP, Calder A, Stocks J, et al. Is chest CT useful in newborn screened infants with cystic fibrosis at 1 year of age? Thorax 2014; 69: 320-327.

19 Duncan JA, Raywood E, Lee S, et al. Improved lung function in preschool children with CF over the last decade. Pediatric Pulmonol 2015; 50: S342.

20 Aurora P, Stanojevic S, Wade A, et al. Lung clearance index at 4 years predicts subsequent lung function in children with cystic fibrosis. Am J Respir Crit Care Med 2011; 183: 752-758.

21 Stanojevic S, Davis SD, Retsch-Bogart G, et al. Progression of lung disease in preschool patients with cystic fibrosis. Am J Respir Crit Care Med 2017; 195: 1216-1225.

22 Davies JC, Wainwright CE, Canny GJ, et al. Efficacy and safety of ivacaftor in patients aged 6 to 11 years with cystic fibrosis with a G551D mutation. Am J Respir Crit Care Med 2013; 187: 1219-1225.

23 Linnane BM, Hall GL, Nolan G, et al. Lung function in infants with cystic fibrosis diagnosed by newborn screening. Am J Respir Crit Care Med 2008; 178: 1238-1244.

24 Jones M, Castile R, Davis S, et al. Forced expiratory flows and volumes in infants. Normative data and lung growth. Am J Respir Crit Care Med 2000; 161: 353-359.

25 Bush A, Sly PD. Evolution of cystic fibrosis lung function in the early years. Curr Opin Pulm Med 2015; 21: $602-608$.

26 Ramsey KA, Ranganathan S, Park J, et al. Early respiratory infection is associated with reduced spirometry in children with cystic fibrosis. Am J Respir Crit Care Med 2014; 190: 1111-1116.

27 Davis SD, Ratjen F, Brumback LC, et al. Infant lung function tests as endpoints in the ISIS multicenter clinical trial in cystic fibrosis. J Cyst Fibros 2016; 15: 386-391. 